\title{
The dietary compound curcumin inhibits p300 histone acetyltransferase activity and prevents heart failure in rats
}

\author{
Tatsuya Morimoto, ${ }^{1}$ Yoichi Sunagawa, ${ }^{1}$ Teruhisa Kawamura, ${ }^{1}$ Tomohide Takaya, ${ }^{1,2}$ \\ Hiromichi Wada, ${ }^{1}$ Atsushi Nagasawa, ${ }^{3}$ Masashi Komeda, ${ }^{3}$ Masatoshi Fujita, ${ }^{4}$ \\ Akira Shimatsu, ${ }^{5}$ Toru Kita, $^{2}$ and Koji Hasegawa ${ }^{1}$
}

\begin{abstract}
1Division of Translational Research, Kyoto Medical Center, National Hospital Organization, Kyoto, Japan. ${ }^{2}$ Department of Cardiovascular Medicine, ${ }^{3}$ Department of Cardiovascular Surgery, and ${ }^{4}$ Department of Human Health Sciences, Graduate School of Medicine, Kyoto University, Kyoto, Japan. ${ }^{5}$ Clinical Research Institute, Kyoto Medical Center, National Hospital Organization, Kyoto, Japan.
\end{abstract}

\begin{abstract}
Hemodynamic overload in the heart can trigger maladaptive hypertrophy of cardiomyocytes. A key signaling event in this process is nuclear acetylation by histone deacetylases and $\mathrm{p} 300$, an intrinsic histone acetyltransferase (HAT). It has been previously shown that curcumin, a polyphenol responsible for the yellow color of the spice turmeric, possesses HAT inhibitory activity with specificity for the p300/CREB-binding protein. We found that curcumin inhibited the hypertrophy-induced acetylation and DNA-binding abilities of GATA4, a hypertrophy-responsive transcription factor, in rat cardiomyocytes. Curcumin also disrupted the p300/GATA4 complex and repressed agonist- and p300-induced hypertrophic responses in these cells. Both the acetylated form of GATA4 and the relative levels of the p300/GATA4 complex markedly increased in rat hypertensive hearts in vivo. The effects of curcumin were examined in vivo in 2 different heart failure models: hypertensive heart disease in salt-sensitive Dahl rats and surgically induced myocardial infarction in rats. In both models, curcumin prevented deterioration of systolic function and heart failure-induced increases in both myocardial wall thickness and diameter. From these results, we conclude that inhibition of p300 HAT activity by the nontoxic dietary compound curcumin may provide a novel therapeutic strategy for heart failure in humans.
\end{abstract}

\section{Introduction}

Heart failure remains one of the leading causes of death in industrialized countries $(1,2)$. In response to an increase in pressure or volume overload, cardiomyocytes undergo hypertrophy, a compensatory response to increased wall stress required to maintain normal cardiac output $(3,4)$. However, hypertrophy is associated with the diastolic dysfunction of the heart and with a significant increase in the risk for sudden death, and it eventually leads to systolic dysfunction or decompensated heart failure (3-5). This process is accompanied by activation of various neurohormonal factors, such as angiotensin II, endothelin-1 (ET-1), and catecholamines (6-7). While present pharmacological therapy for heart failure targets such extracellular molecules, mortality due to heart failure is still high $(2,6)$. To establish a more effective therapeutic strategy for heart failure, it will be necessary to target a common downstream pathway within cardiomyocytes.

Neurohormonal factors bind to myocardial cell-surface receptors and activate a number of subcellular signaling pathways. These signals finally reach nuclei of cardiomyocytes and activate a subset of hypertrophy-responsive transcription factors (8). These include serum response factors; myocyte enhancer factor-2 (9); and a zinc finger protein, GATA4 $(10,11)$. Activation of these transcription factors is mediated, in part, through

Nonstandard abbreviations used: ANF, atrial natriuretic factor; BNP, B-type natriuretic peptide; DR, salt-resistant Dahl; DS, salt-sensitive Dahl; ET-1, endothelin-1; FS, fractional shortening; HAT, histone acetyltransferase; LVEDD, LV end-diastolic dimension; MHC, myosin heavy chain; MI, myocardial infarction; PE, phenylephrine; TSA, trichostatin A.

Conflict of interest: The authors have declared that no conflict of interest exists. Citation for this article: J. Clin. Invest. 118:868-878 (2008). doi:10.1172/JCI33160. acetylation control by histone deacetylases and an intrinsic histone acetyltransferase (HAT), p300 $(12,13)$. p300 serves as a coactivator of GATA4 and induces expression of genes encoding atrial natriuretic factor (ANF), ET-1, and $\beta$-myosin heavy chain ( $\beta$-MHC) (14-16). By its HAT activity, p300 not only acetylates histone to promote an active chromatin configuration but also is able to acetylate GATA4 and to increase its DNA-binding and transcriptional activities (16). Cardiac overexpression of intact p300 in transgenic mice induces acetylation of GATA4 and myocardial cell hypertrophy and promotes LV remodeling after myocardial infarction (MI) in vivo $(16,17)$. However, overexpression of mutant p300 lacking HAT activity is unable to achieve such effects (17). These findings suggest that nuclear acetylation by p300 is a critical event during myocardial cell hypertrophy. However, a pharmacological heart failure therapy that targets p300 HAT activity has yet to be established.

Although a number of studies regarding such a strategy have been performed, very few HAT inhibitors are known so far. Lys$\mathrm{CoA}$ and $\mathrm{H} 3-\mathrm{CoA}-20$ are synthetic HAT inhibitors that are specific for p300 and for PCAF, respectively. However, these agents are not easily able to permeate cells $(18,19)$. Anacardic acid and garcinol are cell-permeable natural HAT inhibitors without specificity for p300/CBP and PCAF $(20,21)$. It has been reported that a cell-permeable natural compound, curcumin, possesses HAT inhibitory activity with specificity for p300/CBP (22). Curcumin is a polyphenol that is responsible for the yellow color of the spice turmeric and is commonly used for its perceived health benefits.

The goal of this study was to determine whether a natural p300specific HAT inhibitor, curcumin, can be used as a therapeutic agent for heart failure. First, we tested the effects of curcumin on 


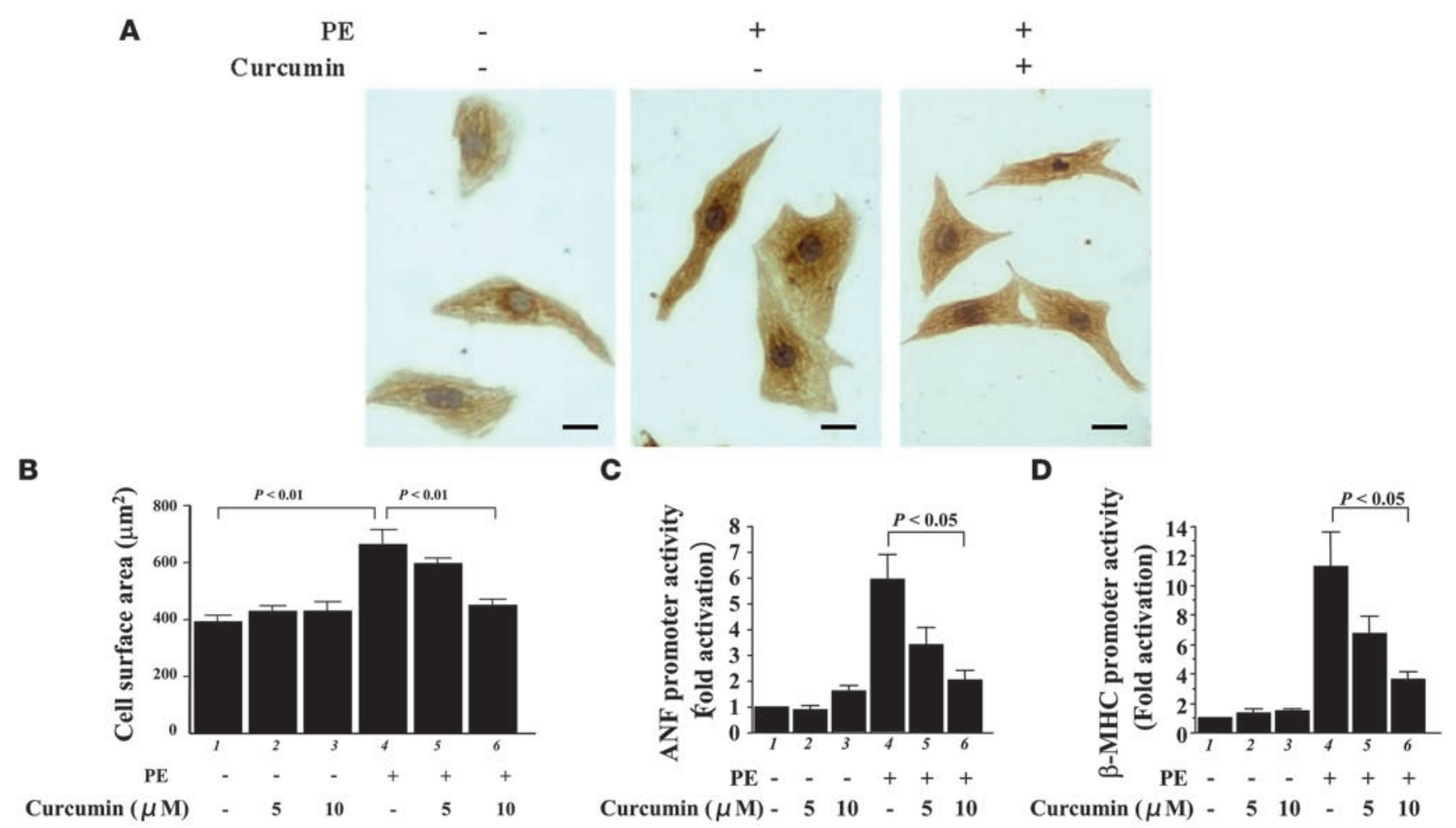

\section{Figure 1}

Curcumin inhibits PE-induced hypertrophic responses in cardiomyocytes. (A and B) Primary cardiomyocytes from neonatal rats were stimulated with saline or $30 \mu \mathrm{M}$ PE in the presence of curcumin (10 $\mu \mathrm{M}$ in $\mathbf{A}$ and 5 or $10 \mu \mathrm{M}$ in $\mathbf{B}$ ) or a corresponding amount of its vehicle (DMSO) as a control for 48 hours. (A) These cells were subjected to immunocytochemistry using the primary antibody against cardiac MHC followed by staining with a secondary antibody conjugated with peroxidase (brown signals). Scale bar: $10 \mu \mathrm{m}$. (B) Myocardial cell-surface area was measured as described in Methods. Values in each group are mean \pm SEM $(\mu \mathrm{m})$ from 50 cells. (C and D) Cardiomyocytes were transfected with $0.5 \mu \mathrm{g}$ of pANF-luc (C) or p $\beta$-MHCluc (D) and $0.0025 \mu \mathrm{g}$ of pRL-SV40. Then, these cells were stimulated with saline or $30 \mu \mathrm{M}$ PE in the presence of curcumin or a corresponding amount of its vehicle for 48 hours. The relative promoter activities were calculated from the ratio of firefly Luc activity to sea pansy Luc activity. The data shown are mean \pm SEM from 3 independent experiments, each carried out in duplicate.

nuclear acetylation and hypertrophic responses in cultured neonatal rat cardiomyocytes. Then we examined the effects of curcumin in 2 different heart failure models in vivo: one model was hypertensive heart disease in salt-sensitive Dahl (DS) rats, and the other model was MI in rats. We demonstrated that inhibition of p300 HAT activity by curcumin prevented the development of heart failure in both models. Thus, we believe that the present study will provide a novel therapeutic strategy for heart failure that targets a common nuclear pathway in cardiomyocytes.

\section{Results}

Curcumin represses bypertrophic responses in cardiomyocytes. We first examined the effects of curcumin on hypertrophic responses in primary cardiomyocytes prepared from neonatal rats. These cells were stimulated with saline or $30 \mu \mathrm{M}$ phenylephrine (PE), an $\alpha_{1}$-adrenergic agonist, in the presence of curcumin $(5$ or $10 \mu \mathrm{M})$ or a corresponding amount of its solvent, DMSO. After 48 hours, we stained these cells with an antibody against cardiac MHC (Figure 1A). Treatment with curcumin inhibited the PE-induced increase in cell size (myocardial cell-surface area) but did not affect the basal cell size (Figure 1B). We then examined the effects of curcumin by transient transfection experiments on PE-induced activities of promoters for ANF and $\beta$-MHC, upregulated expression of which are well-established markers of myocardial cell hypertrophy. Cur- cumin significantly inhibited the induction of ANF (Figure 1C) and $\beta$-MHC (Figure 1D) promoters. However, curcumin alone did not affect the basal levels of these activities.

We then examined the effects of curcumin on myocardial cell hypertrophy induced by overexpression of $\mathrm{p} 300$. Transfection of an expression vector encoding p300 resulted in a marked increase in the amount of p300 protein (Figure $2 \mathrm{~A}$ ) and a significant increase in the myocardial cell-surface area compared with transfection of $\beta$-gal (Figure 2B). Treatment with curcumin significantly inhibited the p300-induced increase in cell size but did not affect the amount of p300. Furthermore, treatment with curcumin significantly inhibited the p300-induced activities of the ANF (Figure 2C) and $\beta$-MHC (Figure 2D) promoters. Taken together, these data demonstrate that curcumin can selectively suppress the PE- and p300-induced hypertrophic responses in cardiomyocytes.

We also used an HDAC inhibitor, trichostatin A (TSA), to induce nuclear hyperacetylation, and mutant p300 (HATmutp300), which loses its HAT activity but retains the ability to interact with transcription factors. Treatment with TSA and transfection of an expression vector encoding HATmutp300 induced a significant increase in cell size. However, the increase by TSA or HATmutp300 was smaller than that caused by intact p300 (Figure 2E, bars 1, 2, 4, and 6). Curcumin inhibited both TSA- and HATmutp300-induced hypertrophy as well as intact p300-induced hypertrophy (Figure 2E, bars 2-6). 
A

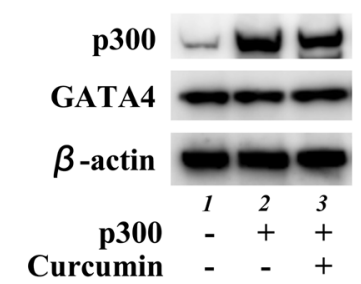

B

D

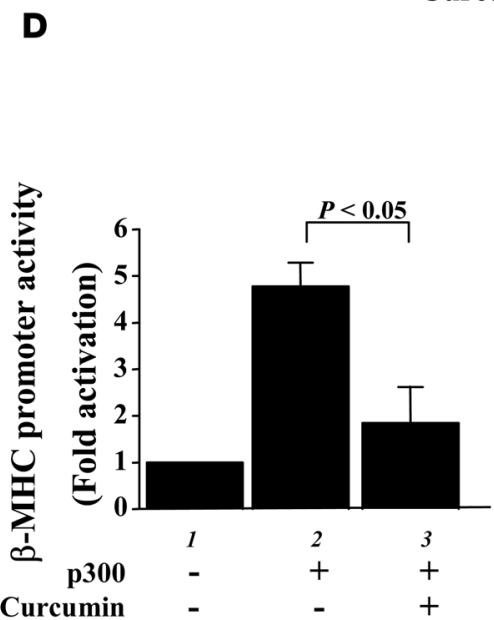

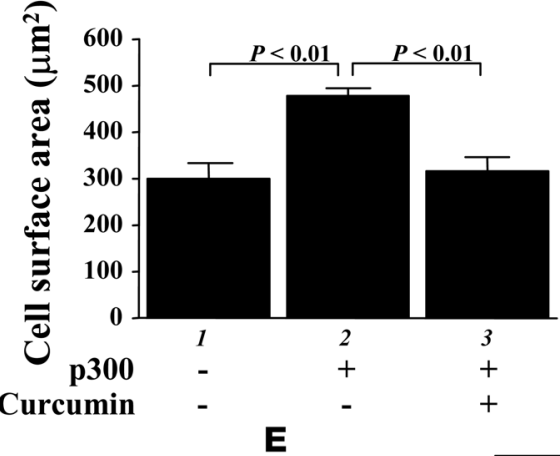

E

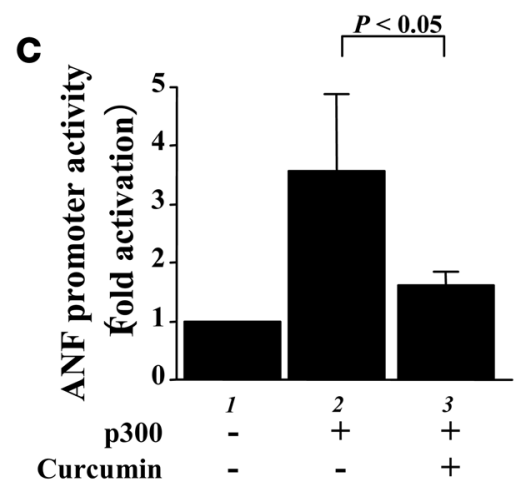



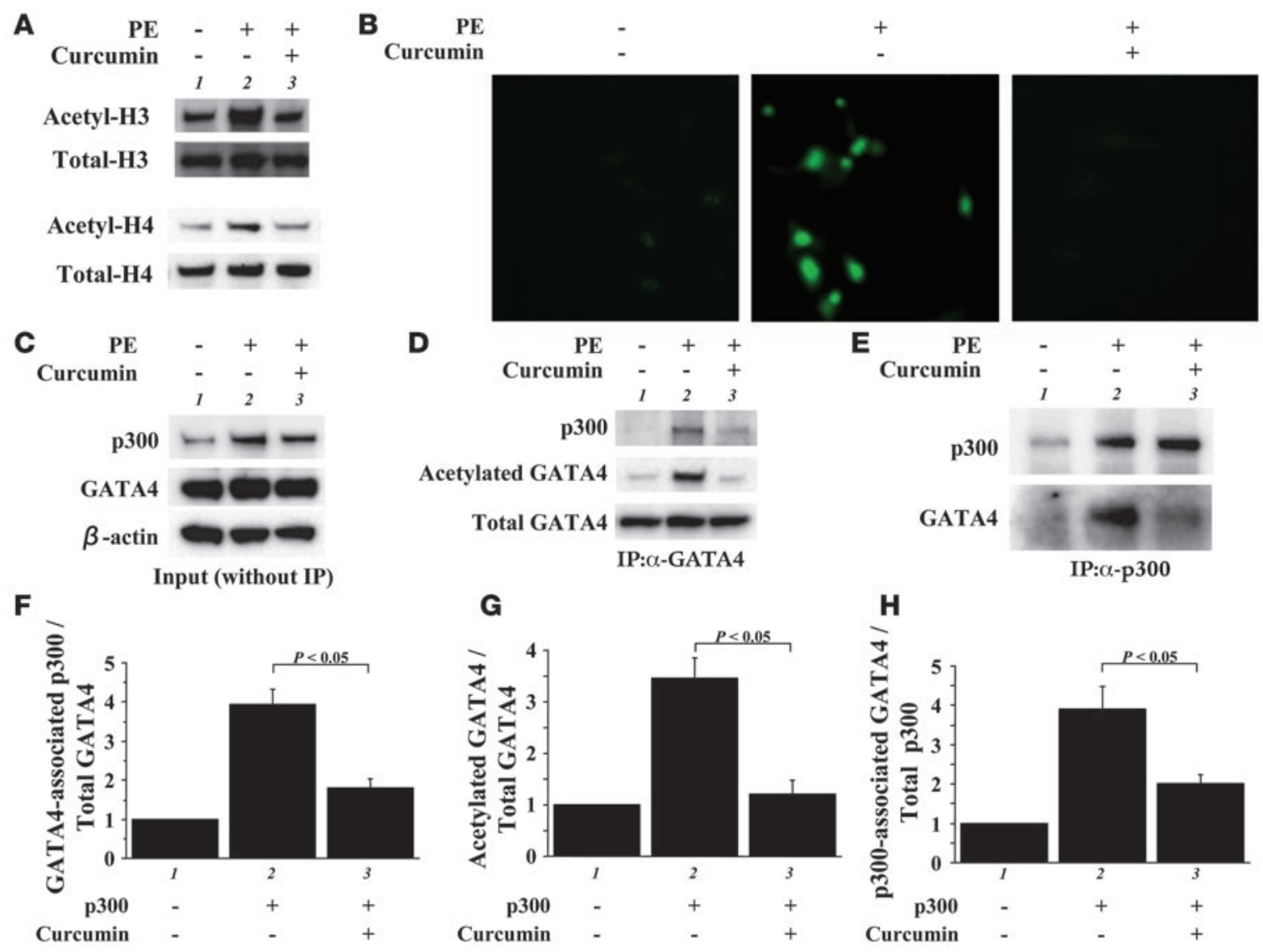

Figure 3

Primary cardiomyocytes from neonatal rats were stimulated with saline (lane 1) or $30 \mu \mathrm{M}$ of PE (lanes 2 and 3 ) in the presence of curcumin (10 $\mu \mathrm{M}$, lane 3) or a corresponding amount of its vehicle (DMSO, lanes 1 and 2) as a control for 48 hours. (A) Proteins isolated by acid extraction from these cells were subjected to Western blotting for acetylated histone-3/4 or total histone-3/4 as indicated. (B) These cells were subjected to indirect immunofluorescence analysis with antibody against acetylated lysine. Original magnification, $\times 200$. (C) Protein extracts from these cells were subjected to Western blotting with anti-GATA4 antibody, anti-p300 antibody, or anti- $\beta$-actin antibody. (D and E) The same extracts (100 $\mu \mathrm{g}$ of protein) were immunoprecipitated with goat anti-GATA4 polyclonal antibody (D) or rabbit anti-p300 polyclonal antibody (E) and subjected to sequential Western blotting with anti-acetylated lysine antibody, anti-p300 antibody, and anti-GATA4 antibody. (F-H) The amounts of GATA4associated p300/total GATA4 binding (F), acetylated GATA4/total GATA4 (G), and p300-associated GATA4/total p300 binding (H) were quantified by densitometry with the use of Multi Gauge V3.0 (FUJIFILM). The data shown are mean \pm SEM from 3 independent experiments.

induced increase in GATA4/DNA binding (Figure 4, A and C, bars $1-3)$. Sp-1/DNA binding was similar among these 3 groups (Figure 4B). These findings demonstrate that curcumin inhibits binding of p300 to GATA4 and represses PE-induced acetylation and DNA binding of GATA4.

Curcumin prevents the development of hypertension-induced heart failure. To determine whether curcumin can prevent the development of heart failure in vivo, we utilized a salt-sensitive Dahl (DS) rat model of hypertension. In this model, LV concentric hypertrophy with preserved systolic function at the age of 11 weeks is followed by a decrease in systolic function at the age of 17-18 weeks. We performed physiological studies on 11-week-old DS rats $(n=39)$ and control normotensive salt-resistant Dahl rats (DR rats, $n=6$ ). Then these rats were randomly assigned to daily oral treatment with curcumin or vehicle ( $1 \%$ gum arabic). Before treatment, there were no differences between the curcumin and vehicle groups in pretreatment data, including body weight, blood pressure, heart rate, LV dimensions, fractional shortening (FS), and wall thickness. Preliminary experiments suggested that $50 \mathrm{mg} / \mathrm{kg} / \mathrm{d}$ of curcumin is sufficient to exert its beneficial effect. Then, we evaluated whether administering $50 \mathrm{mg} / \mathrm{kg} / \mathrm{d}$ of curcumin can preserve LV function. At the age of 18 weeks ( 7 weeks after curcumin or vehicle treatment), we performed physiological studies in all of the surviving rats ( 3 rats in each DR group, 8 rats in the vehicle-treated DS group, and 6 rats in the curcumin-treated DS group). LV systolic function represented by FS was much higher in the curcumin-treated group than in the vehicle-treated group (Figure 5, A, C, and H). Wall thickness was significantly smaller in the curcumin-treated group than in the vehicle-treated group (Figure 5, D and I). Meanwhile, curcumin did not alter FS or wall thickness in DR rats (Figure 5, H and I). LV end-diastolic dimension (LVEDD), body weight, blood pressure, and heart rate did not differ between the curcumin- and vehicle-treated groups of DS rats (Figure 5, B, E, F, and G).

After physiological studies, we measured plasma and LV mRNA levels of B-type natriuretic peptide (BNP), a biochemical marker of heart failure. LV mRNA levels of BNP were higher in DS rats than DR rats in vehicle-treated groups (Figure 6A). In DS rats, these lev- 
A

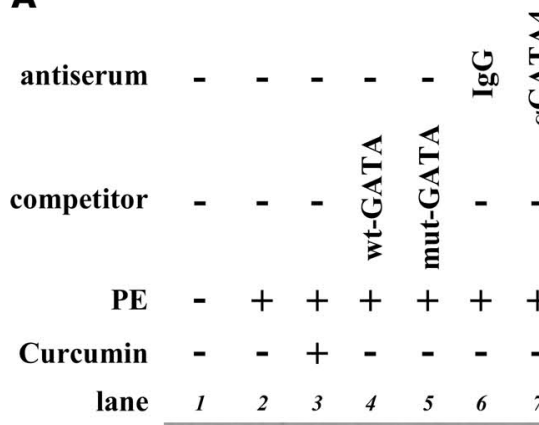

B

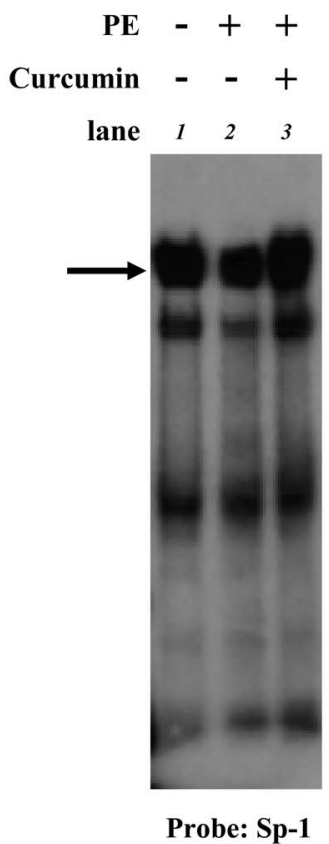

Probe: Sp-1
C

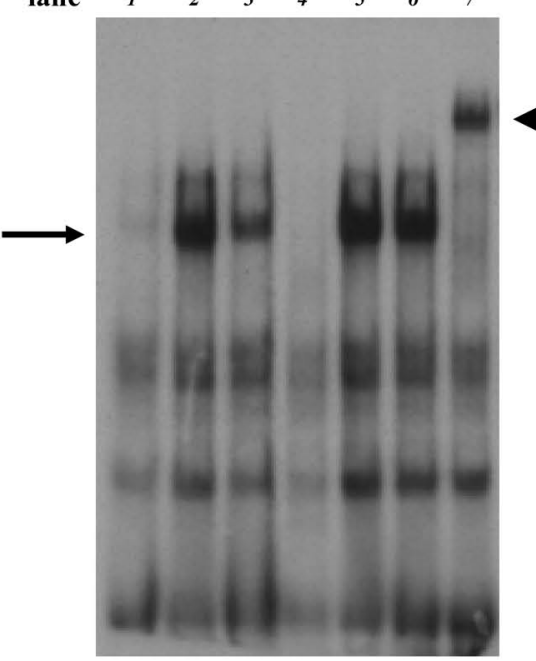

Probe: ET-1-GATA

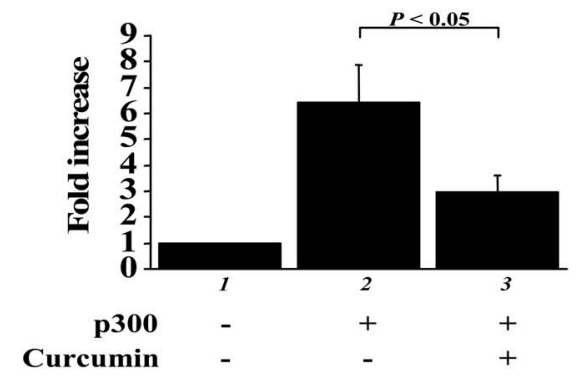

Figure 4

Curcumin represses PE-induced increase in GATA4-DNA binding. (A and B) Primary cardiomyocytes from neonatal rats were stimulated with saline (lane 1) or $30 \mu \mathrm{M}$ of PE (lanes 2 and 3 ) in the presence of curcumin (10 $\mu \mathrm{M}$, lane 3 ) or a corresponding amount of its vehicle (DMSO, lanes 1 and 2) as a control for 48 hours. The nuclear extracts from these cells were probed with a radiolabeled double-stranded oligonucleotide containing the ET-1 GATA site (A) and with one containing the Sp-1 site (B). (C) The amount of GATA4/DNA binding (indicated by a long or short arrow) was quantified by densitometry with the use of NIH Image 1.61. The data shown are mean \pm SEM from 3 independent experiments.

els were significantly higher in the vehicle-treated group than the curcumin-treated group. Consistent with these results, treatment with curcumin significantly inhibited increase in plasma BNP levels in DS rats (Figure 6B). Histological analysis demonstrated that cross-sectional myocardial cell diameter was larger in DS rats than in DR rats in vehicle-treated groups. Curcumin almost completely inhibited hypertension-induced increase in myocardial cell diameter (Figure 6, C and D). In vehicle-treated groups, the amount of fibrosis in perivascular areas was markedly increased in DS compared with DR rats. Curcumin significantly but not completely inhibited the increase in perivascular fibrosis (Figure 6, E and F).

Curcumin inhibits bypertension-induced acetylation of GATA4 in Dabl rats. To determine whether treatment with curcumin affects hypertension-induced acetylation of GATA4 in Dahl rats, we performed immunoprecipitation/Western blotting in LV nuclear extracts from 18-week-old DS and control normotensive DR rats. Before immunoprecipitation, Western blotting demonstrated that p300 levels were increased in DS compared with DR rats, while GATA4 levels were similar in DR and DS rats (Figure 7A). Then, these extracts were subjected to immunoprecipitation with anti-GATA4 antibody, followed by sequential Western blotting with anti-acetylated lysine antibody and anti-p300 antibody.
LV complex containing p300 and GATA4 was increased in DS compared with DR rats (Figure 7B, bottom lanes). In accord with this, the acetylated form of GATA4 increased in DS compared with DR rats (Figure 7B, top lanes). Notably, in DS rats, administration of curcumin decreased the acetylated form of GATA4 (Figure 7D, top lanes) and p300/GATA4 complex (Figure 7D, bottom lanes), while it did not change the expression of GATA4 or p300 themselves (Figure 7C). Quantitative analysis revealed that curcumin decreased by $60 \%$ the ratio of the acetylated form of GATA4 to total GATA4 (Figure 7E). Thus, curcumin inhibited the hypertension-induced acetylation of GATA4 as well as p300/ GATA4 complex in the LV of Dahl rats.

Curcumin prevents the deterioration of LV systolic function after MI. To determine whether the beneficial effect of curcumin is limited to a model of hypertension-induced heart failure or generalized to other types of heart failure, we examined the effect of curcumin on LV remodeling after MI. Fifty-four rats were subjected to MI $(n=40)$ or sham operation $(n=14)$. One week later, we performed physiological studies in all surviving rats. We excluded from this study 2 MI rats whose FS was more than $40 \%$ before randomization. Then, the remaining rats (MI: $n=29$, sham operation: $n=14$ ) were randomly assigned to oral chronic 
A

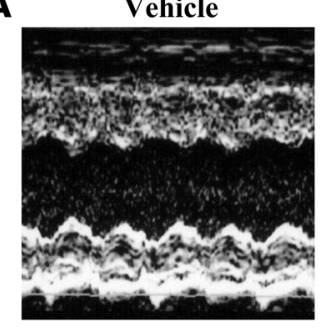

B

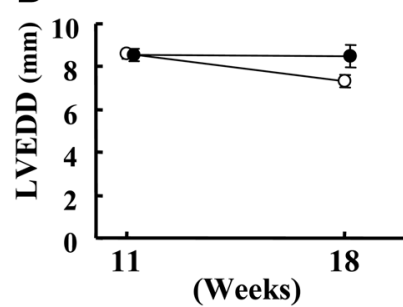

E
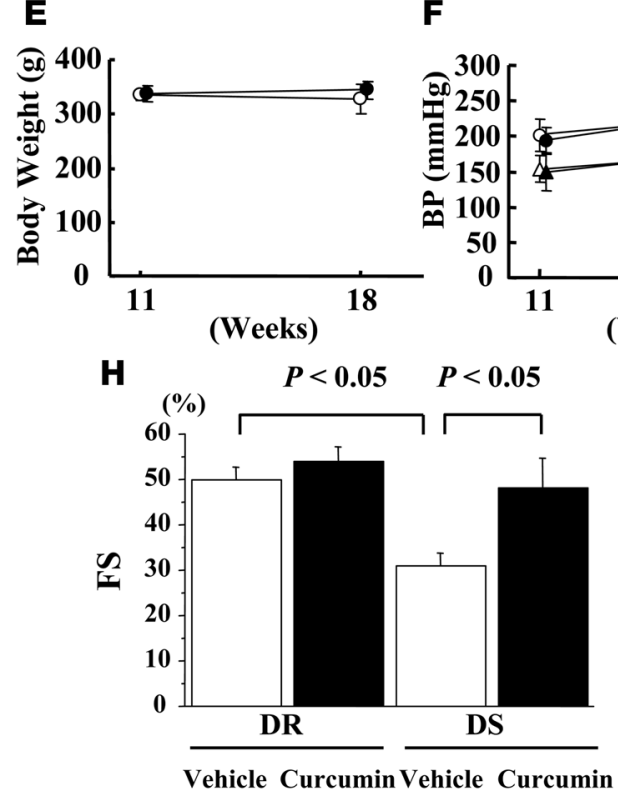

Curcumin

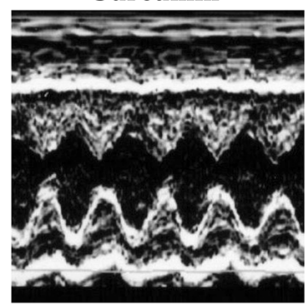

C

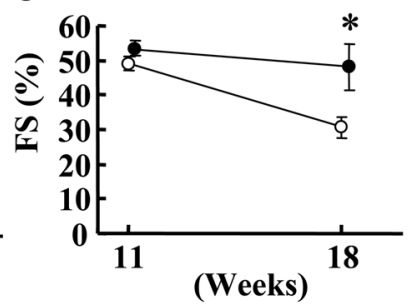

F

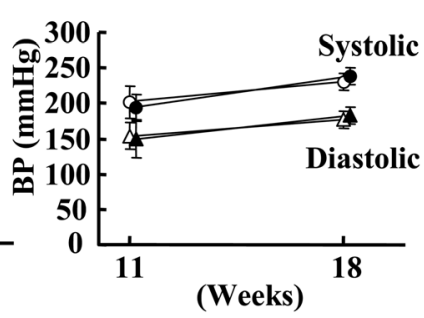

\section{D}

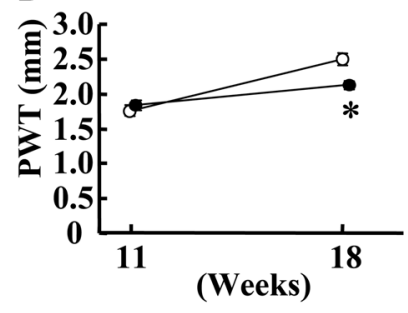

G

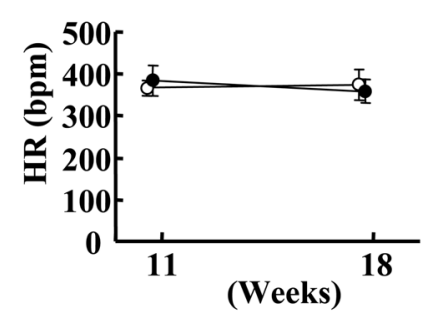

Figure 5

DS rats at the age of 11 weeks were randomly assigned to daily oral treatment with curcumin $(50 \mathrm{mg} / \mathrm{kg} / \mathrm{d})$ or vehicle $(1 \%$ gum arabic) for 7 weeks. Echocardiographic studies were performed at the age of 11 (before treatment) and 18 weeks (after treatment). (A) Representative photographs of M-mode images from vehicleand curcumin-treated $D S$ rats at the age of 18 weeks. (B-G) Values are mean \pm SEM from 6 rats in the curcumin group and 8 rats in the vehicle group. ${ }^{\star} P<0.05$ vs. vehicle group. (B) LVEDD. (C) FS. (D) PWT, posterior wall thickness. (E) Body weight. (F) BP. (G) HR, heart rate. ( $\mathbf{H}$ and $\mathbf{I})$ Values are mean \pm SEM from 3 rats in each DR group, 8 rats in the vehicletreated DS group, and 6 rats in the curcumin-treated DS group.

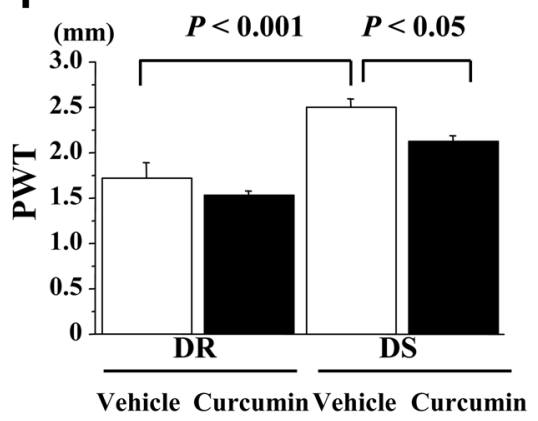

treatment with curcumin $(50 \mathrm{mg} / \mathrm{kg} / \mathrm{d})$ or vehicle $(1 \%$ gum arabic). Before treatment, there were no differences between the curcumin and vehicle groups for any of the data examined, including body weight, blood pressure, heart rate, LV dimensions, FS, and wall thickness. There was no death during the treatment with curcumin or vehicle for 6 weeks. At 7 weeks after MI (6 weeks after the treatment), LV systolic function (represented by FS) was much higher in the curcumin-treated group than in the vehicle-treated group (Figure 8, A, C, and H). Wall thickness was significantly lower in the curcumin-treated group than in the vehicle-treated group (Figure 8, D and I). However, curcumin did not alter FS or wall thickness in sham-operated rats (Figure 8, $\mathrm{H}$ and I). LVEDD, body weight, blood pressure, and heart rate did not differ between the curcumin- and vehicle-treated groups (Figure 8, B, E, F, and G). Histological analysis demonstrated that cross-sectional myocardial cell diameter was larger in MI rats than in sham-operated rats in the vehicle-treated group. Curcumin almost completely inhibited the MI-induced increase in myocardial cell diameter (Figure 8J).

\section{Discussion}

Accumulating evidence suggests that nuclear acetylation controlled by HAT and histone deacetylases is a critical event during pathological cardiomyocyte hypertrophy $(12,13)$. An intrinsic HAT, p300, is able to acetylate histone and hypertrophy-responsive transcription factors such as serum response factor, myocyte enhancer factor-2, and GATA4. Activation by p300 is required not only for pathological myocyte growth but also for normal myocardial development and differentiation. p300-knockout mice die between days 9 and 11.5 of gestation and show reduced expression of muscle structural proteins as well as cardiac structural defects and reduced trabeculation (23). A gene knock-in approach demonstrated that the HAT domain of p300 is essential for heart formation (24). In contrast to myocyte overgrowth, antitumor agent doxorubicin markedly reduces p300 protein levels in cardiomyocytes and induces their apoptosis (25). However, the present study demonstrated that the acetylated form of GATA4 and p300/GATA4 complex were markedly increased in hypertensive hearts in vivo as well as hypertrophied cardio- 
A
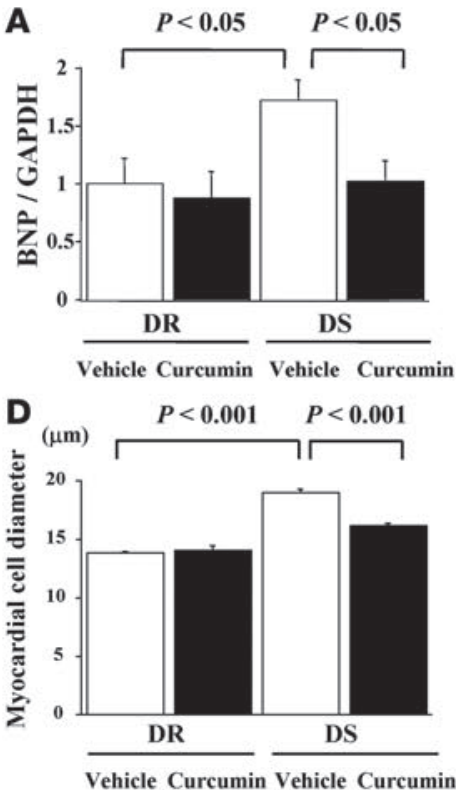

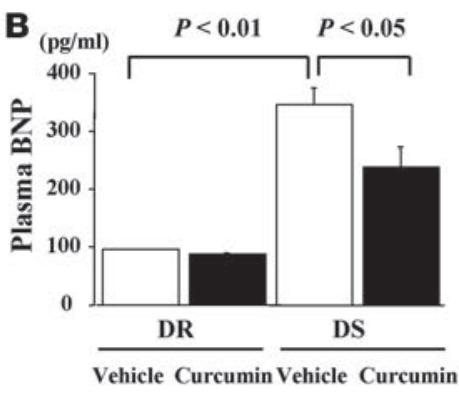

$\mathbf{E}$

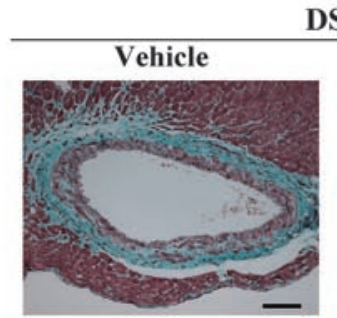

DS

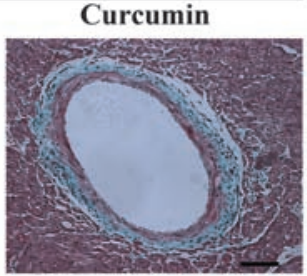

C

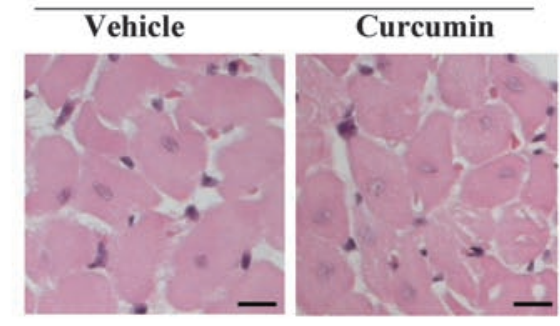

$\mathbf{F}$

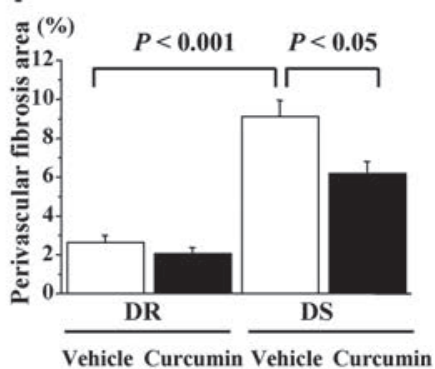

Figure 6

DS and DR rats were treated with curcumin $(50 \mathrm{mg} / \mathrm{kg} / \mathrm{d})$ or vehicle (1\% gum arabic) for 7 weeks. Then, plasma BNP levels were measured (B), and left ventricles were subjected to real-time PCR analysis for BNP and GAPDH (A) and to histological analysis (C-F). (C) Representative photographs of H\&E-stained sections of LV myocardium from vehicle- and curcumin-treated DS rats. Magnification, $\times 400$. Scale bar: $10 \mu \mathrm{m}$. (D) Myocardial cell diameters in left ventricles were measured for at least 50 cells in each animal. (E) Representative photographs of Masson trichrome-stained sections of LV myocardium from vehicle- and curcumin-treated DS rats. Original magnification, $\times 200$. Scale bar: $20 \mu \mathrm{m}$. (F) Areas of perivascular fibrosis in left ventricles were measured for at least 10 intramyocardial coronary arteries in each animal. Results in A, B, D, and F are expressed as mean \pm SEM from 3 rats in each DR group, 8 rats in the vehicle-treated DS group, and 6 rats in the curcumin-treated DS group.

myocytes in culture. These findings suggest that cardiac p300 activity is increased in common types of heart failure in which pathological cardiomyocyte overgrowth occurs in response to hemodynamic overload.

A natural compound, curcumin, repressed agonist- and p300induced hypertrophic responses in cultured neonatal cardiomyocytes. Curcumin inhibited acetylation of histones and GATA4 nearly to the control level. These findings suggest that curcuminmediated inhibition of nuclear acetylation mainly contributes to the repression of myocardial cell hypertrophy. Interestingly, curcumin also disrupted the p300/GATA4 complex formation in cardiomyocytes. It has been reported that $\mathrm{p} 300$, by its HAT activity, is able to induce autoacetylation of p300 (26) and its significant conformational change and dissociate p300 from mediator complexes (27). Paradoxically, curcumin strongly inhibits binding of p300 to mediator complexes (27). This effect of curcumin appears to be independent of its inhibitory action on p300 HAT activity, since curcumin also inhibits binding of catalytically inactive p300 mutant to mediators. Our finding that curcumin disrupts p300/GATA4 complex formation is compatible with this report. At present, the precise mechanisms by which curcumin inhibits binding of p300 to GATA4 in cardiomyocytes are unclear. One possible explanation would be that curcumin induces a p300 allosteric transition that prevents its binding to GATA4. At any event, curcumin may repress hypertrophic responses in cardiomyocytes through at least 2 mechanisms: (a) inhibiting acetylation of histones and hypertrophy-responsive transcription factors; and (b) disrupting the p300/GATA4 complex by a mechanism distinct from inhibiting p300 HAT activity.
We showed that curcumin inhibited HATmutp300- and TSAas well as intact p300-induced hypertrophy. The smaller extent of hypertrophy brought about by TSA or HATmutp300 than that by intact p300 suggests that both the bridging function and HAT activity of p300 are required for maximal hypertrophy. Further studies are needed to clarify to what extent the inhibition of p300 HAT activity and/or disruption of the p300/GATA4 complex contribute to the inhibitory effects of curcumin on hypertrophy induced by PE in culture or via hypertension or MI in animal models. However, our data suggest that both of the 2 mechanisms are significantly involved in the curcumin-mediated inhibition of hypertrophic responses in cardiomyocytes.

Myocardial cell hypertrophy is associated with systolic and diastolic dysfunction of the heart. The present study demonstrated that in 2 different models of heart failure, hypertension and MI, curcumin prevented deterioration of systolic function without affecting systemic hemodynamics. In both models, curcumin significantly decreased LV wall thickness and the diameter of each myocyte. Curcumin-mediated inhibition of cardiomyocyte hypertrophy was also documented by reduced gene expression of BNP, one of the markers of myocardial cell hypertrophy (28). While curcumin significantly reduced perivascular fibrosis as well, the reduction was minimal. Strikingly, curcumin reduced the wall thickness and myocyte diameter of MI rats nearly to the level of those of sham-operated rats. Furthermore, curcumin treatment inhibited the hypertensioninduced acetylation of GATA4 and formation of p300/GATA4 complex in DS rats, in accord with the data in cultured neonatal cardiomyocytes. These findings suggest that curcumin acts 

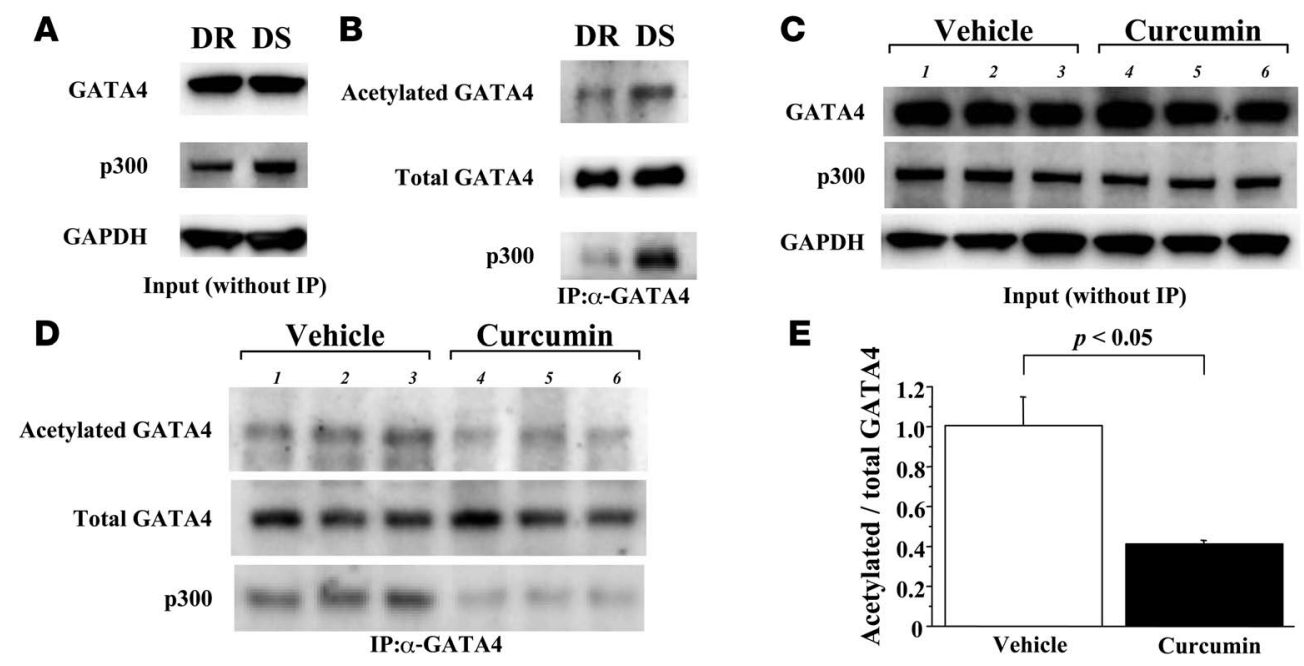

Figure 7

Curcumin inhibits hypertension-induced acetylation of GATA4 as well as p300/GATA4 complex in the LVs of Dahl rats. (A) Protein extracts from the hearts of DR and DS rats at the age of 18 weeks were subjected to Western blotting with anti-p300 antibody, anti-GATA4 antibody, or anti-GAPDH antibody. (B) These extracts were then immunoprecipitated with goat anti-GATA4 polyclonal antibody and subjected to sequential Western blotting with anti-acetylated lysine antibody, anti-p300 antibody, and anti-GATA4 antibody. (C and D) Protein extracts from the hearts of DS rats treated with curcumin $(50 \mathrm{mg} / \mathrm{kg} / \mathrm{d})$ or vehicle ( $1 \%$ gum arabic) for 7 weeks were subjected to Western blotting for p300, GATA4, and GAPDH (C) and then immunoprecipitated with anti-GATA4 antibody followed by sequential Western blotting for acetylated lysine, GATA4, and p300 (D). (E) The levels of signals for acetylated GATA4 and those for total GATA4 were quantified. Results are expressed as mean \pm SEM from 8 vehicle-treated rats and 6 curcumin-treated rats.

directly on the heart and that reversal of myocyte hypertrophy is the main mechanism by which curcumin improves LV systolic function in these rat heart failure models.

As a HAT inhibitor, curcumin is able to inhibit the p300-mediated acetylation of p53 in vitro and in vivo (22). Furthermore, curcumin can disrupt the conformation of the $\mathrm{p} 53$ protein required for its serine phosphorylation. Recently, Sano et al. reported that sustained pressure overload induces an accumulation of p53 that inhibits Hif-1 activity (29). This inhibition results in the impairment of cardiac angiogenesis and in the development of heart failure. Therefore, another possible explanation for the beneficial effect by curcumin might be induction of angiogenesis by inhibiting cardiac p53 activity. In addition to its inhibitory action on p300 HAT activity, curcumin inhibits transcriptional activation by NF-кB (30), a pivotal intracellular mediator of the inflammatory response. NF- $\kappa \mathrm{B}$ signaling is also involved in cardiomyocyte hypertrophy (31). The contribution of this action of curcumin to the improvement of systolic function in hemodynamic overload-induced heart failure should be further investigated. However, inhibition of NF-кB may be important in such human disease states as myocarditis, in which inflammatory processes play a key role in the development of heart failure (32). Curcumin also acts as a free radical scavenger and antioxidant, inhibiting lipid peroxidation and oxidative DNA damage. Puvanakrishnan and colleagues reported that curcumin played a protective role against isoproterenol-induced myocardial necrosis in rats and that this protective effect is attributed to its antioxidant properties (33). These multiple actions of curcumin may be beneficial when clinically applying this agent to patients with heart failure.

As curcumin has potent antiproliferative effects against a variety of cancer cells in vitro and in vivo, 7 clinical trials regarding neoplasms are now ongoing, mainly targeting colon cancer (34). Other clinical trials utilizing curcumin are also underway, including trials for chronic psoriasis vulgaris and Alzheimer disease. The phase I-II human clinical trials have revealed that oral administration of curcumin at doses up to $10 \mathrm{~g} / \mathrm{d}$ is safe. This dosage exceeds the dosage used in this study $(50 \mathrm{mg} / \mathrm{kg} / \mathrm{d})$. Hypertension and MI-induced heart failure are common diseases in industrial countries and predispose patients to circulatory failure and sudden death. Conventional pharmacological therapy such as angiotensin blockers (converting enzyme inhibitors and/or receptor blockers) targets extracellular neurohormonal factors. We believe that the use of curcumin, which targets nuclear signaling pathways in cardiomyocytes, will provide a novel therapeutic strategy against heart failure. Future application of this nontoxic dietary natural compound as a therapeutic agent for heart failure in humans would be particularly interesting.

\section{Methods}

Immunocytochemistry and measurement of cell-surface area. Primary neonatal rat ventricular cardiomyocytes were prepared as previously described $(16,37)$. The cardiomyocytes were grown in flask-style chambers with glass slides (Nalgen Nunc) and stained for cardiac MHC using the indirect immunoperoxidase method, as previously described (35). A total of 40 myocardial fibers were selected randomly from cardiomyocytes stained with anti- $\beta$-MHC antibody that reacts with both $\alpha$ - and cardiac $\beta$-MHC. Then, the surface area of these cells was measured semiautomatically with the aid of an image analyzer (Image Pro-Plus; MediaCybernetics) as described previously (35).

Plasmid constructs. The expression vectors pCMV $\beta$-gal, pCMVwtp300, and pCMVHATmutp300 contain the cytomegalovirus promoter/enhancer fused to cDNA encoding $\beta$-gal, a full-length human p300, and a mutant p300, respectively, in which double amino acid substitutions were introduced in the HAT domain. The plasmids pCMVwtp300 and pCMVHATmutp300 were a gift from Richard Eckner, University of Medicine and Dentistry of New Jersey, Newark, New Jersey, USA. The plasmid constructs pANF-luc and $\mathrm{p} \beta$-MHCluc 

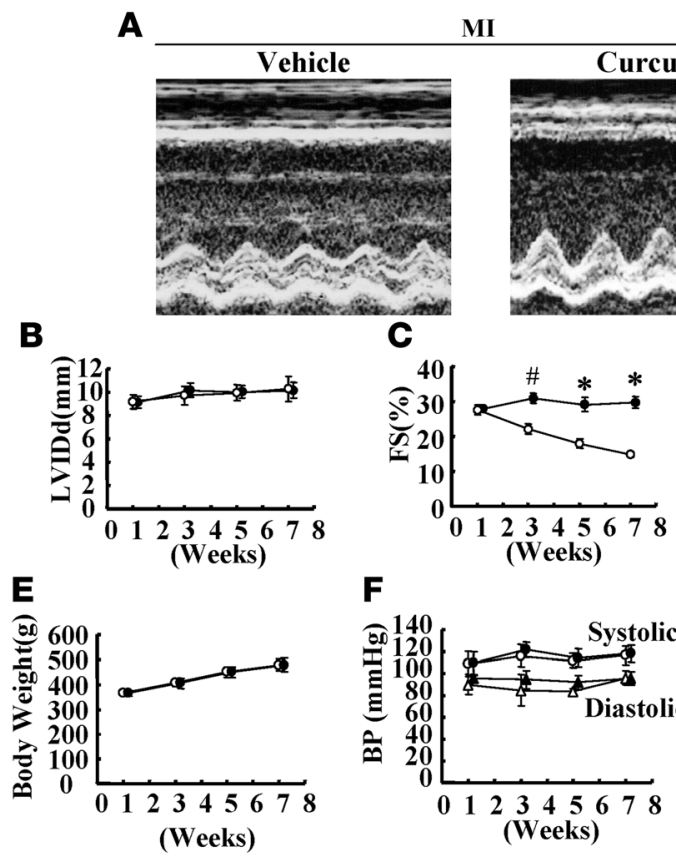

$\mathbf{F}$

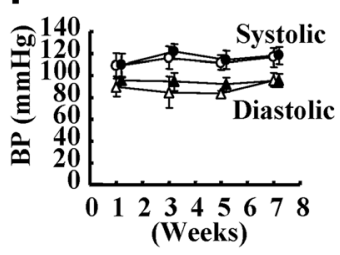

D

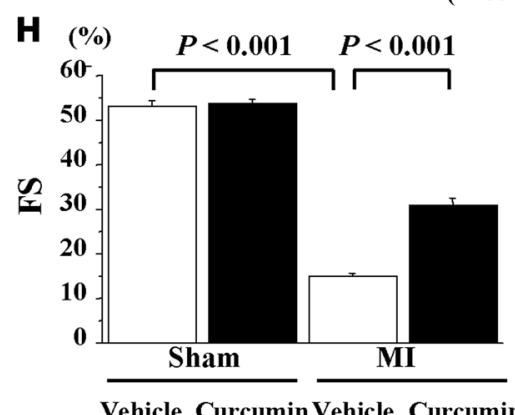

Vehicle Curcumin Vehicle Curcumin

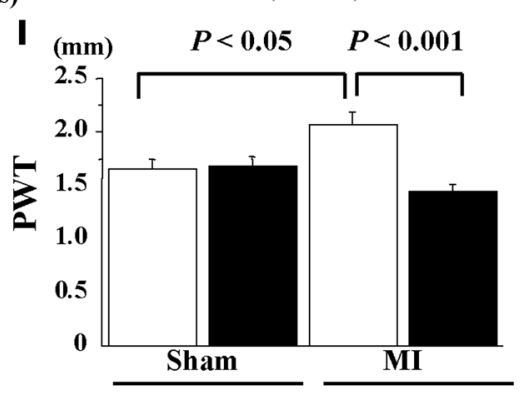

Vehicle Curcumin Vehicle Curcumin

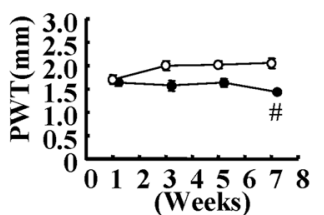

G

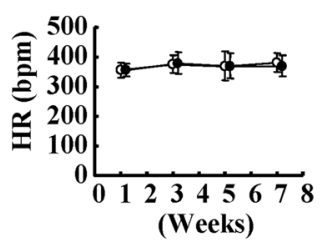

\section{Figure 8}

One week after the operation, rats with MI $(n=29)$ and those that underwent sham operation $(n=14)$ were treated with curcumin $(50 \mathrm{mg} / \mathrm{kg} / \mathrm{d})$ or vehicle (1\% gum arabic) for 6 weeks. Echocardiographic studies were performed every 2 weeks. (A) Representative photographs of M-mode images from vehicle- and curcumin-treated MI rats at 7 weeks after the operation. (B-G) Values are mean \pm SEM from 14 rats in the curcumin group and 15 rats in the vehicle group. ${ }^{\#} P<0.005$ vs. vehicle group; ${ }^{*} P<0.0001$ vs. vehicle group. (B) LVEDD. (C) FS. (D) Diastolic PWT. (E) Body weight. (F) BP. (G) HR. (H-J) Results are expressed as mean \pm SEM from 7 rats in each sham-operated group, 15 rats in the vehicle-treated MI group, and 14 rats in the curcumin-treated Ml group. (J) Myocardial cell diameters in left ventricles were measured for at least 50 cells in each animal.

were firefly luciferase reporter plasmids driven by a 131-bp rat ANF promoter sequence (15) and $333 \mathrm{bp}$ of the rat $\beta$-MHC gene (35), respectively. pRL-SV40, which contains the sea pansy Luc cDNA driven by a simian virus 40 promoter, was purchased from TOYO B-Net Co.

Transfection and dual luciferase assays. Cardiomyocytes were cotransfected with the indicated amounts of DNA using Lipofectamine Plus (Invitrogen) according to the manufacturer's recommendations. After a 2-hour incubation with DNA-Lipofectamine complex, the cells were washed twice with serum-free medium and further incubated for 48 hours with appropriate agents in serum-free medium. The cells were then washed twice with icecold PBS and lysed with lysis buffer, and the activities of firefly and sea pansy Luc were determined in the same cell lysate using a PicaGene Dual kit (TOYO B-Net Co.). The relative promoter activities were calculated from the ratio of firefly Luc to sea pansy Luc.

Detection of histone acetylation. Histones were isolated by acid extraction using a commercial kit (Upstate) according to the manufacturer's recommendations as previously described (36). Western blotting for acetylated histone-3/4 and for total histone-3/4 was performed using goat anti- acetylated histone-3/4 polyclonal antibodies (Santa Cruz Biotechnology Inc.) and rabbit anti-histone-3/4 polyclonal antibodies (Santa Cruz Biotechnology Inc.), respectively.

Detection of nuclear acetylation by immunofluorescence. The staining for acetylated lysine was performed using the indirect immunofluorescence method. The cells were fixed with Bouin solution for 10 minutes at room temperature and subsequently autoclaved for 10 minutes at $121^{\circ} \mathrm{C}$ in $10 \mathrm{mM}$ citrate, $\mathrm{pH}$ 6.0. Cells were incubated with anti-acetylated lysine polyclonal antibody (Cell Signaling) at a dilution of 1:30. The signals were detected with anti-mouse FITC-conjugated secondary antibody at a dilution of 1:75 for 45 minutes.

Analysis of the acetylation state of GATA4 and Western blotting. Immunoprecipitation and Western blotting for acetylated lysine and GATA4 were performed as previously described $(16,17)$. We used goat anti-GATA4 polyclonal antibody (Santa Cruz Biotechnology Inc.) for immunoprecipitation and rabbit polyclonal antibody against acetylated lysine (Cell Signaling), rabbit anti-GATA4 polyclonal antibody, rabbit antip300 polyclonal antibody (Santa Cruz Biotechnology Inc.), mouse anti$\beta$-actin monoclonal antibody (Sigma-Aldrich), and mouse anti-GAPDH 
monoclonal antibody (Molecular Probes; Invitrogen) for Western blotting. The levels of signals were estimated by photographing them using LAS-1000 Plus (FUJIFILM) and quantified using Multi Gauge V3.0 (FUJIFILM).

EMSAs. EMSAs were carried out as previously described using the GATA4 site in the rat ET-1 promoter as a probe $(16,17,37)$. We also used a doublestranded oligonucleotide containing the Sp-1 binding site (Santa Cruz Biotechnology Inc.) as a control probe. The levels of the signals were quantified by densitometry using NIH Image 1.61 (http://rsb.info.nih.gov).

Animals. All procedures were performed in conformity with the Guide for the Care and Use of Laboratory Animals by the Institute of Laboratory Animals, Graduate School of Medicine, Kyoto University, and the protocol was approved by the ethics committee of the Graduate School of Medicine, Kyoto University.

DS rats. Male DS and DR rats were purchased from Japan SLC Inc. After weaning, the rats were fed a $0.3 \% \mathrm{NaCl}$ (low-salt) diet until the age of 6 weeks, after which they were fed an $8 \% \mathrm{NaCl}$ (high-salt) diet. Eleven-weekold DS and DR rats were randomly assigned to treatment with either curcumin (Wako Japan) or vehicle (1\% gum arabic). All drugs were given to these rats orally by gastric gavage once a day. Animals were monitored, and body weights and in vivo blood pressures were measured weekly.

MI rats. MI was induced in male rats weighing $250-290 \mathrm{~g}$ by proximal ligation of the left anterior descending coronary artery through left thoracotomy, as previously described (38). Rats were anesthetized with $1.0 \%-1.5 \%$ isoflurane. After coronary ligation, ST elevation on electrocardiography and a color change in the LV myocardium were observed in all rats. The same surgical procedure was performed in a sham-operated group of rats, except that the suture around the coronary artery was not tied. Animals were monitored, and body weights were measured weekly. Serial echocardiography and measurement of in vivo blood pressures were performed biweekly.

Transthoracic echocardiography. Cardiac function was noninvasively evaluated by echocardiography according to a method previously described (38). In brief, images were recorded using a 10- to $12-\mathrm{MHz}$ phased-array transducer (model 21380A with HP SONOS 5500 imaging system; Agilent Technologies). LVEDD and end-systolic dimension (LVESD) were measured with M-mode tracings from the short-axis view of the LV at the papillary muscle level. Percent FS was calculated as follows: \%FS = [(LVEDD - LVESD) / LVEDD] × 100. All measurements were performed in a blinded fashion according to the guidelines of the American Society for Echocardiology and averaged over 3 consecutive cardiac cycles.

Histological analysis. After physiological studies, all surviving rats were euthanized, and their hearts were removed. The excised hearts were cut into 2 transverse slices at the mid-level of the papillary muscles; the specimens were fixed in $10 \%$ buffered formalin and embedded in paraffin, after which 4- $\mu \mathrm{m}$-thick sections were stained with H\&E and Masson trichrome. Quantitative assessments of cross-sectional myocardial cell diameter and perivascular fibrotic area were performed on 20 randomly chosen highpower fields in each section with the use of an Axioskop 2 FS plus (Zeiss).

Measurement of plasma BNP and LV BNP $m R N A$. Blood samples were obtained from all surviving rats, and plasma BNP concentrations were determined using a radioimmunoassay kit (Peninsula Lab.). Measurement of LV BNP mRNA was performed by real-time RT-PCR analysis. Total RNA was isolated from left ventricles using TRIzOL reagent (Invitrogen). RNA samples were treated with DNaseI (Invitrogen) to eliminate genomic DNA contamination, and cDNA was synthesized using SuperScriptTMII reverse transcriptase (Invitrogen). For real-time PCR, the reaction was performed with SYBR Green PCR master mix (Applied Biosystems), and the products were analyzed with a thermal cycler (ABI Prism 7900HT sequence detection system). Levels of GAPDH transcript were used to normalize cDNA levels. Rat BNP-specific PCR primers were: sense, 5'-TTCCGGATCCAGGAGAGACTT-3'; antisense, 5'-CCTAAAACAACCTCAGCCCGT-3'.

Statistics. Data are presented as mean \pm SEM. Statistical comparisons were performed with the use of unpaired 2-tailed Student $t$ tests or ANOVA with Scheffe's test where appropriate, with a probability value less than 0.05 taken to indicate significance.

\section{Acknowledgments}

We thank S. Nagata, M. Nishikawa, S. Shibata, and N. Chiba for excellent technical assistance. This work was supported in part by a Grant-in-Aid for Scientific Research from the Ministry of Education, Culture, Sports, Science, and Technology of Japan to K. Hasegawa and T. Morimoto and by grants to T. Morimoto from the Mishima Kaiun Memorial Foundation, Kowa Life Science Foundation, and Kampou Science Foundation.

Received for publication July 2, 2007, and accepted in revised form November 28, 2007.

Address correspondence to: Koji Hasegawa, Division of Translational Research, Kyoto Medical Center, National Hospital Organization, 1-1 Mukaihata-cho Fukakusa, Fushimi-ku, Kyoto 6128555, Japan. Phone: 81-75-641-9161; Fax: 81-75-641-9252; E-mail: koj@kuhp.kyoto-u.ac.jp.
1. Ho, K.K., Levy, D., Kannel, W.B., and Pinsky, J.L. 1993. The epidemiology of heart failure: the Framingham study. J. Am. Coll. Cardiol. 22:6-13.

2. Dominguez, L.J., Parrinello, G., Amato, P., and Licata, G. 1999. Trends of congestive heart failure epidemiology: contrast with clinical trial results. Cardiologia. 44:801-808.

3. Dorn, G.W., II, Robbins, J., and Sugden, P.H. 2003. Phenotyping hypertrophy: eschew obfuscation. Circ. Res. 92:1171-1175.

4. Frey, N., and Olson, E.N. 2003. Cardiac hypertrophy: the good, the bad, and the ugly. Annu. Rev. Physiol. 65:45-79.

5. Chien, K.R. 1999. Stress pathways and heart failure. Cell. 98:555-558.

6. Lohse, M.J., Engelhardt, S., and Eschenhagen, T. 2003. What is the role of $\beta$-adrenergic signaling in heart failure? Circ. Res. 93:896-906.

7. Molkentin, J.D., and Dorn, I.G., II. 2001. Cytoplasmic signaling pathways that regulate cardiac hypertrophy. Annu. Rev. Physiol. 63:391-426.

8. Chien, K.R., et al. 1993. Transcriptional regulation during cardiac growth and development. Annu. Rev.
Physiol. 55:77-95.

9. Kolodziejczyk, S.M., et al. 1999. MEF2 is upregulated during cardiac hypertrophy and is required for normal post-natal growth of the myocardium. Curr. Biol. 9:1203-1206.

10. Herzig, T.C., et al. 1997. Angiotensin II type Ia receptor gene expression in the heart: AP-1 and GATA-4 participate in the response to pressure overload. Proc. Natl. Acad. Sci. U. S. A. 94:7543-7548.

11. Hasegawa, K., Lee, S.J., Jobe, S.M., Markham, B.E., and Kitsis, R.N. 1997. cis-Acting sequences that mediate induction of beta-myosin heavy chain gene expression during left ventricular hypertrophy due to aortic constriction. Circulation. 96:3943-3953.

12. Backs, J., and Olson, E.N. 2006. Control of cardiac growth by histone acetylation/deacetylation. Circ. Res. 98:15-24.

13. McKinsey, T.A., and Olson, E.N. 2004. Cardiac histone acetylation-therapeutic opportunities abound. Trends Genet. 20:206-213.

14. Dai, Y.S., and Markham, B.E. 2001. p300 Functions as a coactivator of transcription factor GATA-4. J. Biol. Chem. 276:37178-37185.
15. Kakita, T., et al. 1999. p300 protein as a coactivator of GATA-5 in the transcription of cardiacrestricted atrial natriuretic factor gene. J. Biol. Chem. 274:34096-34102.

16. Yanazume, T., et al. 2003. Cardiac p300 is involved in myocyte growth with decompensated heart failure. Mol. Cell. Biol. 23:3593-3606.

17. Miyamoto, S., et al. 2006. Histone acetyltransferase activity of p300 is required for the promotion of left ventricular remodeling after myocardial infarction in adult mice in vivo. Circulation. 113:679-690.

18. Lau, O.D., et al. 2000. HATs off: selective synthetic inhibitors of the histone acetyltransferases p300 and PCAF. Mol. Cell. 5:589-595.

19. Cebrat, M., Kim, C.M., Thompson, P.R., Daugherty, M., and Cole, P.A. 2003. Synthesis and analysis of potential prodrugs of coenzyme A analogues for the inhibition of the histone acetyltransferase p300. Bioorg. Med. Chem. 11:3307-3313.

20. Balasubramanyam, K., Swaminathan, V., Ranganathan, A., and Kundu, T.K. 2003. Small molecule modulators of histone acetyltransferase p300. J. Biol. Chem. 278:19134-19140. 
21. Balasubramanyam, K., et al. 2004. Polyisoprenylated benzophenone, garcinol, a natural histone acetyltransferase inhibitor, represses chromatin transcription and alters global gene expression. J. Biol. Chem. 279:33716-33726.

22. Balasubramanyam, K., et al. 2004. Curcumin, a novel p300/CREB-binding protein-specific inhibitor of acetyltransferase, represses the acetylation of histone/nonhistone proteins and histone acetyltransferase-dependent chromatin transcription. J. Biol. Chem. 279:51163-51171.

23. Yao, T.P., et al. 1998. Gene dosage-dependent embryonic development and proliferation defects in mice lacking the transcriptional integrator p300. Cell. 93:361-372.

24. Shikama, N., et al. 2003. Essential function of p300 acetyltransferase activity in heart, lung and small intestine formation. EMBO J. 22:5175-5185.

25. Kawamura, T., et al. 2004. Expression of p300 protects cardiac myocytes from apoptosis in vivo. Biochem. Biophys. Res. Commun. 315:733-738.

26. Thompson, P.R., et al. 2004. Regulation of the p300 HAT domain via a novel activation loop. Nat. Struct.
Mol. Biol. 11:308-315.

27. Black, J.C., Choi, J.E., Lombardo, S.R., and Carey, M. 2006. A mechanism for coordinating chromatin modification and preinitiation complex assembly. Mol. Cell. 23:809-818.

28. Gardner, D.G. 2003. Natriuretic peptides: markers or modulators of cardiac hypertrophy? Trends Endocrinol. Metab. 14:411-416.

29. Sano, M., et al. 2007. p53-induced inhibition of Hif- 1 causes cardiac dysfunction during pressure overload. Nature. 446:444-448.

30. Aggarwal, B.B., and Shishodia, S. 2006. Molecular targets of dietary agents for prevention and therapy of cancer. Biochem. Pharmacol. 71:1397-1421.

31. Purcell, N.H., et al. 2001. Activation of NF-kappa $B$ is required for hypertrophic growth of primary rat neonatal ventricular cardiomyocytes. Proc. Natl. Acad. Sci. U. S. A. 98:6668-6673.

32. Alter, P., Rupp, H., and Maisch, B. 2006. Activated nuclear transcription factor kappaB in patients with myocarditis and dilated cardiomyopathy - relation to inflammation and cardiac function. Biochem. Biophys. Res. Commun. 339:180-187.
33. Manikandan, P., et al. 2004. Curcumin modulates free radical quenching in myocardial ischemia in rats. Int. J. Biochem. Cell Biol. 36:1967-1980.

34. Sharma, R.A., et al. 2001. Pharmacodynamic and pharmacokinetic study of oral Curcuma extract in patients with colorectal cancer. Clin. Cancer Res. 7:1894-1900.

35. Hirai, M., et al. 2004. FOG-2 competes with GATA-4 for transcriptional coactivator $\mathrm{p} 300$ and represses hypertrophic responses in cardiac myocytes. J. Biol. Chem. 279:37640-37650.

36. Kawamura, T., et al. 2005. Acetylation of GATA4 is involved in the differentiation of embryonic stem cells into cardiac myocytes. J. Biol. Chem. 280:19682-19688.

37. Morimoto, T., et al. 2000. Phosphorylation of GATA- 4 is involved in $\alpha 1$-adrenergic agonistresponsive transcription of the Endothelin-1 gene in cardiac myocytes. J. Biol. Chem. 275:13721-13726.

38. Tambara, K., et al. 2003. Transplanted skeletal myoblasts can fully replace the infarcted myocardium when they survive in the host in large numbers. Circulation. 108(Suppl. 1):II259-II263. 